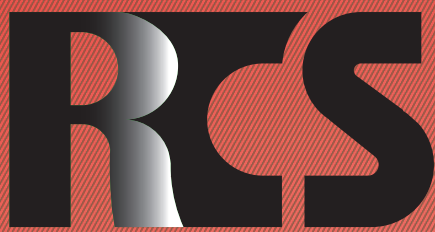

Depósito legal ppi $201502 Z U 4662$

Esta publicación científica en formato digital es continuidad de la revista impresa Depósito Legal: pp $197402 Z$ U789

- ISSN: 1315-9518 • ISSN-E: 2477-9431

Revista de Ciencias Sociales

Universidad del Zulia. Revista de la Facultad de Ciencias Económicas y Sociales Vol. XXVII,

No. 4,2021

Esta publicación científica en formato digital es continuidad de la revista impresa Depósito Legal: pp $197402 Z U 789$ ISSN: $1315-9518$ 


\title{
Desempeño de Universidades y Escuelas de Negocios: Instituciones españolas presenciales y a Distancia
}

\author{
Barrientos Oradini, Nicolás Pablo* \\ Vicencio-Ríos, Gustavo** \\ Armijos Armijos, Juan Carlos ${ }^{* * *}$ \\ González Navarro, Yuri Christian ${ }^{* * * *}$
}

\section{Resumen}

En materia educativa se asiste a una clara evolución en las metodologías empleadas como consecuencia de la progresiva integración de las nuevas tecnologías de la Información y la Comunicación. En ese sentido, el objetivo de la presente investigación consiste en analizar el desempeño administrativo y financiero de universidades y escuelas de negocio existentes en el mercado español bajo la modalidad a distancia y educación virtual, buscando responder el siguiente interrogante: ¿Es posible, mediante un análisis de desempeño financiero de las Universidades y Escuelas de Negocio en España, estudiar el comportamiento de las instituciones comparando públicas y privadas; presenciales y virtuales; considerando además, los principales rankings internacionales que puntúan a las instituciones de educación superior? Se trata de un trabajo analítico de tipo cualitativo, cuyo método de investigación histórica es el analítico-sintético. Entre los resultados se evidencia una primacía económica financiera y en los rankings de las instituciones privadas por sobre las públicas y de las modalidades mixtas por sobre la meramente presencial y la virtual. Se concluye, que el trabajo prueba que un buen desempeño financiero es una condición necesaria pero no suficiente para sobresalir en materia de calidad.

Palabras clave: Nuevas Tecnologías de la Información y la Comunicación; educación a distancia; educación presencial; universidad; escuela de negocios.

Doctor Philosophiae en Administración de Empresas. Doctor of Philosophy in Business Administration. Académico en la Universidad Andrés Bello, Chile. E-mail: nicolas.barrientos@unab.cl iD ORCID: https://orcid.org/0000-0002-8973-8647

** PhD. en Administración de Empresas. Profesor Asociado en la Universidad Diego Portales, Chile. E-mail: gustavo.vicencio@udp.cl (iD) ORCID: https://orcid.org/0000-0003-2408-8804

**** Magister en Control de Gestión. Académico Asistente de la Universidad Santo Tomás, Chile. E-mail: jarmijos@santotomas.cl ORCID: https://orcid.org/0000-0002-0618-1987

***** Magister en Contabilidad y Auditoría de Gestión. Docente en la Universidad Autónoma de Chile, Chile. E-mail: yuri.gonzalez@uautonoma.cl (iD ORCID: https://orcid.org/0000-0003-4735-6920

Recibido: 2021-06-19 · Aceptado: 2021-09-03 


\title{
Performance of Universities and Business Schools: Face-to-face and Distance Spanish Institutions
}

\begin{abstract}
In educational matters, there is a clear evolution in the methodologies used as a consequence of the progressive integration of the new Information and Communication technologies. In this sense, the objective of this research is to analyze the administrative and financial performance of existing universities and business schools in the Spanish market under the distance and virtual education modality, seeking to answer the following question: Is it possible, through a analysis of financial performance of Universities and Business Schools in Spain, study the behavior of institutions comparing public and private; face-to-face and virtual; considering also the main international rankings that score higher education institutions? It is a qualitative analytical work, whose historical research method is analytical-synthetic. Among the results, an economic and financial primacy is evidenced, and in the rankings of private institutions over public institutions and of mixed modalities over merely face-to-face and virtual. It is concluded that the work proves that good financial performance is a necessary but not sufficient condition to excel in terms of quality.
\end{abstract}

Keywords: New Information and Communication Technologies; long distance education; face-to-face education; University; business School.

\section{Introducción}

Al reflexionar respecto de cómo se enseña y se aprende en la actualidad y como se hacía en el pasado, puede observarse que se ha producido una clara evolución en las metodologías empleadas. Dicho progreso responde principalmente a un cambio producido a raíz de la progresiva integración de las nuevas tecnologías de la llamada Sociedad de la Información y la Comunicación, impactando en nuevas modalidades y vínculos de enseñanza-aprendizaje. Una consecuencia directa del salto tecnológico ha sido la emergencia de la Educación a Distancia a lo largo del siglo XXI, dando lugar tanto al Blended Learning (modalidad mixta), y al E-learning (online) (Araya-Castillo, 2015), siendo esta última el centro de este trabajo.

A nivel mundial, dadas las condiciones actuales de la sociedad, la educación a distancia o virtual, es una modalidad educativa que cada día suma más adeptos, encontrándose como una tendencia en pleno auge. El principal motivo de esta tendencia educativa se basa principalmente en que la educación presencial no responde a los requerimientos actuales, puesto que por ejemplo los estudiantes no pueden asistir con regularidad a su institución educativa, existiendo problemas para compatibilizar horarios con su jornada laboral. A estas cuestiones estructurales se agrega otra coyuntural signada por una situación anómala como una pandemia mundial, la cual logró reforzar la importancia que tiene la actualización y acompañamiento tecnológico en la formación educativa.

En España, por ejemplo, existen en el presente, dos universidades públicas que imparten sus cursos a distancia, las cuales, al estar integradas dentro del sistema nacional de Educación Superior, buscan competir al nivel de las tradicionales, es decir, pretenden ser tan competitivas como las que tienen una oferta 
netamente de cursos presenciales. En primer orden, la Universidad Nacional de Educación a Distancia (UNED), creada en 1972, tiene como objetivo potenciar la igualdad de oportunidades y colaborar para disminuir la sobredemanda de algunas instituciones de educación superior tradicionales. Por otro lado, existe una segunda universidad con modalidad a distancia, la Universitat Oberta de Catalunya (UOC). Esta institución fue creada en 1995 y se fundamenta en el uso de las telecomunicaciones, de modo que el diseño del proceso de enseñanza y aprendizaje se basa en un Campus Virtual.

Teniendo en cuenta este contexto mundial y nacional, el objeto de estudio de la presente investigación es analizar el desempeño económico y financiero de universidades y escuelas de negocio existentes en el mercado español bajo la modalidad a distancia y de la educación virtual, teniendo siempre como referencia a la modalidad presencial. Busca, además, responder la siguiente interrogante: $¿$ Es posible, mediante un análisis de desempeño financiero de las Universidades y Escuelas de Negocio en España, estudiar el comportamiento de las instituciones comparando públicas y privadas; presenciales y virtuales; en función además de los principales rankings internacionales de desempeño para instituciones de educación superior?

Para afrontar dicha pregunta de investigación se esboza la siguiente hipótesis de trabajo: La aplicación de una mayor calidad de la Educación Superior con una adecuada formación de competencias ejecutivas en programas de formación con uso de las nuevas tecnologías de la Información y la Comunicación (TIC) en las escuelas de negocios, será viable una vez que las entidades de Educación Superior establezcan medidas para el mejoramiento de sus programas en base a metas de ahorro, con un desempeño financiero favorable que logre impactar como atributo relevante en la decisión de inversión, generando también beneficios cuantificables para la oferta y la demanda (Bernal, 2010). El caso español es escogido dada la heterogeneidad de modalidades de impartición educativa y la gran cantidad de datos disponibles.

\section{Algunas precisiones conceptuales}

\subsection{E-Learning}

De acuerdo con Area y Adell (2009), el E-Learning o educación en línea se define como la "modalidad de enseñanza-aprendizaje que consiste en el diseño, puesta en práctica y evaluación de un curso formativo a través de redes de computadores" (p.2), introduciendo así grandes diferencias con la formación presencial. Además, el método online se encuentra centrado en el participante, puesto que el estudiante resulta más activo, flexible, autónomo y colaborativo (Cabero, 2004; Maftum y Batista, 2008; Crespo-Fajardo y Pillacela-Chin, 2021).

La característica distintiva es la separación física entre los actores del proceso, aunque una de las diferencias con más repercusiones sería el cambio de roles de estos. En cuanto al docente, este intenta buscar o diseñar recursos para el aprendizaje e integrarlos en el entorno virtual aparte de orientar sus estrategias de EnseñanzaAprendizaje desde la perspectiva online. Respecto al discente o alumno, el nuevo rol se corresponde con adoptar un papel más activo, trabajar de forma colaborativa con los demás y estar dispuesto a emplear recursos de aprendizaje en soportes multimedia.

Al respecto, Sangrá (2001); Varguillas y Bravo (2020); Morán et al. (2021); Hinojosa, Epiquién y Morante (2021); consideran que la principal diferencia entre la educación presencial y la online corresponde al cambio de medio y las cuestiones derivadas de su optimización, tales como el entorno donde tengan lugar las actividades formativas.

En este sentido, puede considerarse que los autores plantean la necesidad de reestructurar los esquemas cognitivos en cuanto a la concepción de las actividades formativas, atendiendo a las características de 
cada una de ellas, pero también en relación con las capacidades de los profesionales que las gestionan al ser indispensable saber adaptarse a las nuevas situaciones que se plantean. Esto haría referencia tanto al contexto académico como al organizativo, dado que en ambos casos el responsable del proceso (profesor, docente o formador), deberá velar por facilitar el aprendizaje a su alumnado incidiendo en otra diferencia clave entre la formación tradicional y el E-learning; la flexibilización del proceso de Enseñanza-Aprendizaje.

Este último aspecto está centrado en el desarrollo de estrategias de aprendizaje colaborativo mediado puesto que, al no haber limitaciones de espacio, se permite llegar a un número más amplio de participantes y -sobre todo en el caso de las organizaciones- mejorar la posibilidad de autogestión del tiempo que favorece en gran medida la realización de las acciones formativas fuera del horario laboral. De este modo, existiría la posibilidad de establecer relaciones diferidas en el tiempo, ya sea tanto vinculada a la comunicación sincrónica -contacto a tiempo real-, como asincrónica -no simultánea- como podría ser a través del correo electrónico (ValverdeBerrocoso, 2002).

Siendo más específicos, resulta relevante referirse a las plataformas de trabajo. Este estudio se focalizará en plataforma VLE (Virtual Learning Environment) o EVA (Entorno Virtual de Aprendizaje), refiriéndose al espacio donde desarrollar la acción formativa y donde se incluyen los contenidos, las actividades o las herramientas de comunicación, entre otros. Por lo tanto, se podría llegar a entender el EVA, como la aplicación del Learning Management System (LMS), aunque también encontraría una última concreción en forma de los Personal Learning Environment (PLE); un entorno de aprendizaje personalizado formado por un conjunto de aplicaciones diversas o bien un software específico con aplicaciones integradas (Gómez, 2017).

Para su optimo provecho, las plataformas de formación virtual deberían velar por la armonía entre ambas partes (profesor-alumno), teniendo en cuenta factores tales como los objetivos propuestos o los medios para alcanzarlos, la gestión y dirección del proceso como grandes condicionantes para elegir la plataforma indicada (Smith, 2001).

Por su parte ¿Qué puede aportar el uso de una plataforma EVA como recurso formativo en las organizaciones? En primer lugar, favorece la construcción del conocimiento desde una visión holística siempre enriquecedora, aludiendo a que los diferentes recursos quedan recogidos en un espacio en el que se puede acceder cuando se desee desde un ordenador o determinados dispositivos móviles. En segundo término, para Martí (2011), su utilización incide en el efecto motivacional para la participación asociada a esta metodología ante lo que se conoce como mera recepción pasiva de información.

Sobre la presencia en las organizaciones, Macau (2004) considera que "las TIC pueden tener varios papeles en el seno de una organización. Más aún, desarrollan varias funciones al mismo tiempo" (p.10), reiterando la importancia de contemplarlas al plantear los objetivos y la estructura organizativa para ser competitivas. Sin embargo, los EVA también presentan ciertas dificultades a la hora de integrarse en su totalidad a la actividad organizativa. Una práctica muy extendida que no contribuye a su calidad pedagógica consiste en centrar el uso de las plataformas sólo en la entrega de contenidos y el uso individual, o bien el E-Reading, dejando de lado todo su potencial como herramienta de comunicación y construcción conjunta del conocimiento.

\subsection{La cuestión de la calidad}

En cuanto a la calidad, actualmente existe gran importancia e impulso desde el mercado hacia la valorización de la calidad en las instituciones de educación superior. Casi en su totalidad, los países cuentan con instituciones que acreditan, certifican y aseguran la calidad de las universidades. Es así como la noción de calidad surge como componente vital en los avances técnicos 
que se quieran implementar en el ámbito educativo. Es por ello, que son integradas por los administradores prácticas como el benchmarking y la gestión en base a resultados (Kells, 1992; Alstete, 1995).

La educación superior vía internet ha tenido un relevante incremento en el último tiempo, facilitando una reducción de costos, pero generando preocupación acerca de cómo validar su calidad (Altbach, Reisberg y Rumbley, 2009). La economía de la educación actual, asume el problema de la calidad como un proceso técnico de organización productiva y racionalización de mercado (Kells, 1992; Alstete, 1995), en que los procesos de modernización han sido endógenamente producidos por las entidades universitarias (cómo lógica adaptativa), generando vinculación activa con el sector privado y el mercado. Estas instituciones participan dentro de un entorno dinámico y complejo (Araya, 2014), debido a la influencia de los mercados globalizados (Denegri et al., 2013).

Lo anteriormente señalado genera importantes cambios en el ámbito educacional (Altbach et al., 2009), fomentando la creación de nuevas instituciones de educación superior (Jain, Sahney y Sinha, 2013), e incentiva el mejoramiento continuo de las actividades que se desarrollan (Dill, 2010). De acuerdo con Brunner (2006), las instituciones de educación superior se enfocan en alcanzar un desempeño óptimo, generar ingresos, y obtener prestigio académico, así como social. El éxito de estas instituciones no solo se mide por los resultados financieros o participación de mercado, sino también por la percepción y satisfacción de los clientes (Jain, Sinha y Sahney, 2011).

Es por ello, por lo que las Instituciones de Educación Superior con una positiva satisfacción de sus estudiantes, contribuyen con la mejora de la calidad y competitividad del sector en que participan (Arambewela y Hall, 2006). Esta situación se encuentra presente en las escuelas de negocios, puesto que para los estudiantes un buen posicionamiento influye al momento de decidir en qué institución estudiar. Tal contexto competitivo obliga a las instituciones a posicionarse de la mejor manera en los diferentes rankings nacionales e internacionales, tales como "América y Economía", "QS Universities Rankings", "Academic Ranking of World Universities".

\subsection{Indicadores financieros}

El último punto por definir en términos teóricos se remite a los indicadores financieros para la gestión institucional. Este tipo de análisis consiste en estudiar los datos contenidos en los Estados Financieros, relacionarlo con otros antecedentes y obtener así información útil para la toma de decisiones. De este modo, existe una serie de herramientas mediante las cuales se puede analizar los estados financieros. Entre los más tradicionales se encuentran:

a. Análisis de ratios o razones: Corresponden a un conjunto de relaciones que se crean a partir de cuentas del balance, del estado de resultados o de ambos. Buscan establecer condiciones de rentabilidad, liquidez y endeudamiento.

b. Análisis vertical: Este análisis busca determinar la estructura de activos, de pasivos y de resultados, así como observar si existen cambios importantes a través del tiempo.

c. Análisis horizontal: Este análisis persigue establecer la evolución porcentual, a través de los años, de cada partida o línea del activo, del pasivo y del estado de resultados. Se emplea en la forma de índice, asignándole el valor 100 al valor más antiguo.

\section{Metodología}

Se trata de un trabajo analítico de tipo cualitativo. La presente investigación, cuenta en primer lugar con la aplicación del método histórico lógico; en este sentido, el método de investigación histórica es analítico-sintético. Esto implica que el análisis es un procedimiento que posibilita descomponer un todo en sus partes y cualidades, en sus múltiples relaciones, propiedades y componentes. De acuerdo con 
Rodríguez y Pérez (2017), este método permite estudiar el comportamiento de cada parte. Por el contrario, la síntesis corresponde al proceso inverso, mediante el cual se construye la unión de las partes analizadas anteriormente, lo que facilita determinar la relación de éstas.

Para realizar dicho proceso fue necesario llevar adelante una actividad previa consistente en la recolección de datos mediante una investigación documental. De acuerdo con Pinto (1992), dicha actividad es el complejo de operaciones que afectan al contenido y a la forma de los documentos originales, para transformarlos en otros documentos representativos de aquellos, que facilitan al usuario su identificación precisa, su recuperación y su difusión.

Con la información disponible, es factible la realización de análisis costobeneficio de las nuevas formas virtuales para una evaluación de los proyectos de inversión en niveles educativos de programas ejecutivos con uso de TIC. Este análisis, corresponde a una evaluación socioeconómica del programa o proyecto, para determinar la conveniencia de inversión mediante la valoración en términos monetarios de los costos y beneficios asociados directa e indirectamente, para la ejecución y operación de las modalidades online en las escuelas de negocio españolas.

Se consideraron en total 17 Universidades (12 privadas y 5 públicas), así como 5 Escuelas de Negocios. De este modo, se procederá a analizar y contrastar las universidades y poder definir su desempeño financiero $^{(1)}$. A tal efecto, para un mejor desarrollo, el estudio se dividirá en cuatro análisis: Universidades privadas versus públicas; universidades presenciales versus presenciales/online versus online; escuelas de negocios presenciales versus presenciales/ online versus online; y, por último, el desempeño financiero que tienen versus su posición en tres rankings definidos ${ }^{(2)}$.

La metodología de este trabajo sería de carácter cualitativo o no tradicional: Se orienta a profundizar casos específicos y no a generalizar. Su preocupación no es prioritariamente medir, sino cualificar y describir el fenómeno social a partir de rasgos determinantes, según sean percibidos por los elementos que están dentro de la situación estudiada (Bernal, 2010).

\section{Resultados y discusión}

\subsection{Análisis de las universidades online, presenciales/online y presenciales}

\section{a. Razón circulante}

Del 2017 al 2018, hubo una mejora en cuanto a la capacidad de generar efectivo por parte de las universidades $100 \%$ online puesto que, en promedio, se obtuvo 1,49 veces el 2017 y 6,51 el 2018, lo cual lleva a pensar que están obteniendo un mejor rendimiento con respecto al pago de sus pasivos de corto plazo. Un escenario no muy favorable para las universidades con modalidad virtual, debido a que poseen un exceso de efectivo dentro de las organizaciones y lleva a deducir que no están aprovechando las oportunidades de inversión para mejorar la infraestructura y el servicio que otorgan (ver Tabla 1).

Tabla 1

Comparación universidades presenciales vs. presencial/online vs. online

\begin{tabular}{lcc}
\hline Universidades & $\mathbf{2 0 1 8}$ & $\mathbf{2 0 1 7}$ \\
& Razón Circulante & Razón Circulante \\
\hline Universidades 100\% online & & \\
Universidad Internacional de la Rioja & 24,73 & 1,01 \\
Universidad a Distancia de Madrid & 2,84 & 2,87 \\
Universitat Oberta de Catalunya & 0,52 & 0,91 \\
Universidad Internacional de Valencia & & 1,41 \\
Universidad Isabel I & 3,50 & 1,83 \\
\hline
\end{tabular}




\section{Cont... Tabla 1}

\begin{tabular}{lcc}
\hline Universidad Nacional de Educación a Distancia & 0,96 & 0,91 \\
Promedio & $\mathbf{6 , 5 1}$ & $\mathbf{1 , 4 9}$ \\
Universidades presenciales / online & & \\
Universidad Europea Miguel de Cervantes & 3,77 & 3,74 \\
Universidad Nebrija & 0,44 & 0,78 \\
Universidad Camilo José Cela & 6,28 & 7,90 \\
Universidad Autónoma de Barcelona & 0,57 & 0,42 \\
Universidad Autónoma de Madrid & 1,89 & 1,70 \\
Promedio & $\mathbf{2 , 5 9}$ & $\mathbf{2 , 9 1}$ \\
Universidades presenciales & & \\
Universidad Alfonso X el Sabio & 15,35 & 14,50 \\
Universidad Católica San Antonio de Murcia & 0,14 & 0,53 \\
Universidad de Alcalá & 3,22 & 2,62 \\
Universidad Pompeu Fabra & 0,61 & 0,24 \\
Universidad Complutense de Madrid & 1,28 & 1,38 \\
Universidad Rey Juan Carlos & 2,29 & 0,83 \\
Promedio & $\mathbf{3 , 8 2}$ & $\mathbf{3 , 3 5}$ \\
\hline
\end{tabular}

Fuente: Elaboración propia, 2021 en base a información publicada por las propias instituciones y datos obtenidos del Sistema de Análisis de Balances Ibéricos (SABI, 2020).

Analizando las universidades con ambas modalidades, se puede decir que hubo una disminución en la capacidad de generar efectivo del 2017 al 2018, debido a que, en promedio, se obtuvo 2,91 veces el 2017 y 2,59 el 2018 (ver Tabla 1). Los resultados siguen siendo números mayores a uno $\mathrm{y}$, por consiguiente, siguen teniendo la capacidad de saldar sus obligaciones de corto plazo.

Por último, las universidades presenciales, al igual que las universidades $100 \%$ online, presentaron un incremento en su capacidad de generar efectivo para pagar sus pasivos de corto plazo, del 2017 al 2018; las presenciales, con un promedio de 3,35 veces y 3,82 veces, respectivamente; y las $100 \%$ online, 1,49 veces en 2017 y 6,51 veces en 2018. Por lo cual, este tipo de universidades no poseen dificultades para cubrir sus pasivos de corto plazo, pero, a su vez, podrían no estar aprovechando el exceso de efectivo que poseen en inversiones que beneficien a largo plazo.

\section{b. Razón deuda total}

Como se puede observar en la Tabla 2, las universidades $100 \%$ online, en promedio, han sido capaces de disminuir su porcentaje de deuda de manera significativa. Por lo que, del 2017 al 2018, están menos comprometidos con los préstamos de terceras personas y, por lo tanto, disminuyen sus probabilidades de quiebra. 
Tabla 2

\begin{tabular}{llc} 
Razón deuda total: Universidades presenciales vs. presencial/online vs. online \\
\hline Universidades & $\begin{array}{l}\text { Razón deuda } \\
\text { total (2018) }\end{array}$ & $\begin{array}{l}\text { Razón deuda } \\
\text { total (2017) }\end{array}$ \\
\hline Universidades 100\% online & 0,99 & 0,96 \\
Universidad Internacional de la Rioja & 0,06 & 0,93 \\
Universidad a Distancia de Madrid & 0,77 & 0,40 \\
Universitat Oberta de Catalunya & & 0,62 \\
Universidad Internacional de Valencia & 0,06 & 0,43 \\
Universidad Isabel I & 0,43 & 0,45 \\
Universidad Nacional de Educación a Distancia & $\mathbf{0 , 4 6}$ & $\mathbf{0 , 6 3}$ \\
Promedio & 0,21 & 0,20 \\
Universidades presenciales / online & 0,72 & 0,73 \\
Universidad Europea Miguel de Cervantes & 0,30 & 0,27 \\
Universidad Nebrija & 0,64 & 0,49 \\
Universidad Camilo José Cela & 0,39 & 0,39 \\
Universidad Autónoma de Barcelona & $\mathbf{0 , 4 5}$ & $\mathbf{0 , 4 2}$ \\
Universidad Autónoma de Madrid & & \\
Promedio & 0,06 & 0,06 \\
Universidades presenciales & 0,99 & 0,99 \\
Universidad Alfonso X el Sabio & 0,17 & 0,12 \\
Universidad Católica San Antonio de Murcia & 0,59 & 0,95 \\
Universidad de Alcalá & 0,67 & 0,69 \\
Universidad Pompeu Fabra & 0,27 & 0,25 \\
Universidad Complutense de Madrid & $\mathbf{0 , 4 5}$ & $\mathbf{0 , 5 1}$ \\
Universidad Rey Juan Carlos & & \\
Promedio & &
\end{tabular}

Fuente: Elaboración propia, 2021 en base a información publicada por las propias instituciones y datos obtenidos del Sistema de Análisis de Balances Ibéricos (SABI, 2020).

En el caso de las universidades con ambas modalidades, en promedio, se ve un leve aumento del porcentaje de deuda de 0,03 puntos porcentuales (ver Tabla 2). Por lo cual, se puede pensar que este tipo de universidades han necesitado una mayor cantidad de financiamiento por parte de terceros para poder operar, resultando un aspecto negativo, aunque no del todo preocupante en esos niveles.

Por último, de acuerdo con las universidades presenciales, en promedio, han disminuido su porcentaje de deuda de 0,51 a 0,45 , del 2017 al 2018. Por lo que, da el indicio que necesitan menos dinero para financiarse con terceros y, por consiguiente, tendrían menos obligaciones de pago con los acreedores.

\section{c. Rotación de activos}

En las universidades $100 \%$ online, la rotación de activos, en promedio, obtuvo una mejoría, pasando de 0,92 a 0,99 (ver Tabla 3 ). Por lo que este tipo de universidades han aumentado la eficiencia con la cual utilizan sus activos totales para generar ventas, es decir, han sido capaces de explotar su efectivo, invertir en activos fijos, mejorar infraestructura online para atraer una mayor cantidad de estudiantes que estén dispuestos a pagar por este tipo de servicios de este tipo de instituciones. 
Tabla 3

Rotación de activos: Universidades presenciales vs. presencial/online vs. online

\begin{tabular}{lll}
\hline Universidades & $\begin{array}{l}\text { Rotación de } \\
\text { activos (2018) }\end{array}$ & $\begin{array}{l}\text { Rotación de } \\
\text { activos (2017) }\end{array}$ \\
\hline $\begin{array}{l}\text { Universidades 100\% online } \\
\text { Universidad Internacional de la Rioja }\end{array}$ & 0,97 & \\
Universidad a Distancia de Madrid & 1,06 & 0,88 \\
Universitat Oberta de Catalunya & 1,26 & 0,98 \\
Universidad Internacional de Valencia & & 1,48 \\
Universidad Isabel I & 0,81 & 0,59 \\
Universidad Nacional de Educación a Distancia & 0,84 & 0,77 \\
Promedio & $\mathbf{0 , 9 9}$ & 0,82 \\
Universidades presenciales / online & & $\mathbf{0 , 9 2}$ \\
Universidad Europea Miguel de Cervantes & 0,83 & \\
Universidad Nebrija & 1,51 & 0,76 \\
Universidad Camilo José Cela & 0,66 & 1,62 \\
Universidad Autónoma de Barcelona & 0,98 & 0,58 \\
Universidad Autónoma de Madrid & 1,15 & 1,01 \\
Promedio & $\mathbf{1 , 0 3}$ & 1,16 \\
Universidades presenciales & $\mathbf{1 , 0 3}$ \\
Universidad Alfonso X el Sabio & 0,21 & \\
Universidad Católica San Antonio de Murcia & 0,00 & 0,21 \\
Universidad de Alcalá & 0,44 & 0,00 \\
Universidad Pompeu Fabra & 0,50 & 0,43 \\
Universidad Complutense de Madrid & 0,24 & 0,58 \\
Universidad Rey Juan Carlos & 0,31 & 0,25 \\
Promedio & $\mathbf{0 , 2 8}$ & 0,33 \\
\hline
\end{tabular}

Fuente: Elaboración propia, 2021 en base a información publicada por las propias instituciones y datos obtenidos del Sistema de Análisis de Balances Ibéricos (SABI, 2020).

En las universidades con ambas modalidades, desde el año 2017 al 2018, el promedio de la rotación de activos se mantuvo constante, puesto que poseen la misma eficiencia al momento de utilizar los activos totales para generar ventas. Por último, las universidades presenciales obtuvieron una leve disminución en la rotación de activos, pasando de 0,30 a 0,28 en los años 2017-2018 (ver Tabla 3). Por lo tanto, han ido perdiendo la eficiencia al momento de utilizar sus activos, es decir, la inversión en estos no ha sido totalmente aprovechada y, por consiguiente, no han generado ventas con el nivel de utilización de activos que poseen en el año 2018.

\section{d. Rentabilidad sobre el patrimonio (ROE)}

Las universidades $100 \%$ online poseen, en promedio, un aumento significativo del retorno para los accionistas, pasando de un $44 \%$ en el 2017 a un $272 \%$ en el 2018 (ver Tabla 4). Por lo cual, se hace más atractivo invertir en este tipo de universidades de acuerdo con el retorno que se podría alcanzar, es decir, desde el 2017 al 2018 ha ido en aumento la utilidad por acción que reciben los accionistas. 
Tabla 4

Rentabilidad sobre el patrimonio: Universidades presenciales vs. presencial/ online vs. online

\begin{tabular}{lll}
\hline Universidades & ROE (2018) & ROE (2017) \\
\hline Universidades 100\% online & & \\
Universidad Internacional de la Rioja & 13,16 & 1,80 \\
Universidad a Distancia de Madrid & 0,002 & 0,005 \\
Universitat Oberta de Catalunya & $-0,01$ & $-0,03$ \\
Universidad Internacional de Valencia & & 0,31 \\
Universidad Isabel I & 0,42 & 0,51 \\
Universidad Nacional de Educación a Distancia & 0,03 & 0,07 \\
Promedio & $\mathbf{2 , 7 2}$ & $\mathbf{0 , 4 4}$ \\
Universidades presenciales / online & & \\
Universidad Europea Miguel de Cervantes & 0,19 & 0,16 \\
Universidad Nebrija & 0,334 & 0,101 \\
Universidad Camilo José Cela & 0,12 & 0,07 \\
Universidad Autónoma de Barcelona & $-0,12$ & 0,01 \\
Universidad Autónoma de Madrid & $-0,01$ & 0,03 \\
Promedio & $\mathbf{0 , 1 0}$ & $\mathbf{0 , 0 7}$ \\
Universidades presenciales & & \\
Universidad Alfonso X el Sabio & 0,05 & 0,05 \\
Universidad Católica San Antonio de Murcia & 3,437 & 0,084 \\
Universidad de Alcalá & 0,04 & 0,06 \\
Universidad Pompeu Fabra & 0,02 & 0,11 \\
Universidad Complutense de Madrid & $-0,01$ & 0,01 \\
Universidad Rey Juan Carlos & 0,02 & 0,06 \\
Promedio & $\mathbf{0 , 5 9}$ & $\mathbf{0 , 0 6}$ \\
\hline
\end{tabular}

Fuente: Elaboración propia, 2021 en base a información publicada por las propias instituciones y datos obtenidos del Sistema de Análisis de Balances Ibéricos (SABI, 2020).

Por su parte, las universidades con ambas modalidades de estudio presentaron, en promedio, una leve mejoría en su retorno para los accionistas, pasando de un $7 \%$ a un $10 \%$ en los años 2017-2018 (ver Tabla 4), lo cual es positivo debido a que este tipo de universidades va aumentando su atractivo financiero, a pesar de los bajos puntos porcentuales en los cuales aumentó.

Al igual que los dos casos anteriores, las universidades presenciales también presentaron una mejora en su rentabilidad del patrimonio puesto que, en promedio, se logró obtener un $59 \%$ en el retorno generado para los accionistas. Por lo que, de un año a otro, las universidades presenciales se vuelven mucho más atractivas que antes en base a la visión de los inversionistas y el mercado financiero.

\section{e. Rentabilidad sobre los activos (ROA)}

Para las universidades con modalidad $100 \%$ online, se presentó un incremento de $4 \%$ en la rentabilidad sobre los activos totales, donde el promedio pasó de $6 \%$ a un $10 \%$ entre los años 2017-2018 (ver Tabla 5). Por lo cual, existe una leve mejoría en la eficiencia con la cual este tipo de universidades utilizan sus activos para generar utilidades. 


\section{Tabla 5 \\ Rentabilidad sobre los activos: Universidades presenciales vs. presencial/online} vs. online

\begin{tabular}{lll}
\hline Universidades & ROA (2018) & ROA (2017) \\
\hline Universidades 100\% online & & \\
Universidad Internacional de la Rioja & 0,14 & 0,08 \\
Universidad a Distancia de Madrid & 0,002 & 0,005 \\
Universitat Oberta de Catalunya & $-0,003$ & $-0,01$ \\
Universidad Internacional de Valencia & & 0,12 \\
Universidad Isabel I & 0,33 & 0,13 \\
Universidad Nacional de Educación a Distancia & 0,02 & 0,04 \\
Promedio & $\mathbf{0 , 1 0}$ & $\mathbf{0 , 0 6}$ \\
Universidades presenciales / online & & \\
Universidad Europea Miguel de Cervantes & 0,15 & 0,13 \\
Universidad Nebrija & 0,092 & 0,027 \\
Universidad Camilo José Cela & 0,086 & 0,05 \\
Universidad Autónoma de Barcelona & $-0,04$ & 0,00 \\
Universidad Autónoma de Madrid & $-0,01$ & 0,03 \\
Promedio & $\mathbf{0 , 0 6}$ & $\mathbf{0 , 0 5}$ \\
Universidades presenciales & & \\
Universidad Alfonso X el Sabio & 0,05 & 0,09 \\
Universidad Católica San Antonio de Murcia & 0,029 & 0,001 \\
Universidad de Alcalá & 0,032 & 0,05 \\
Universidad Pompeu Fabra & 0,01 & 0,005 \\
Universidad Complutense de Madrid & $-0,01$ & 0,01 \\
Universidad Rey Juan Carlos & 0,02 & 0,04 \\
Promedio & $\mathbf{0 , 0 2}$ & $\mathbf{0 , 0 3}$ \\
\hline
\end{tabular}

Fuente: Elaboración propia, 2021 en base a información publicada por las propias instituciones y datos obtenidos del Sistema de Análisis de Balances Ibéricos (SABI, 2020).

De acuerdo con la información de las universidades con ambas modalidades, existió un incremento de un $1 \%$, donde el promedio pasó de 5\% a 6\% desde el año 2017 al 2018, tal como se aprecia en la Tabla 5. Por lo que, al igual que en el caso de las universidades con modalidad virtual, existe una mayor eficiencia al momento de utilizar los activos totales, pero en menor cantidad.

Al contrario de los dos casos anteriores, las universidades presenciales han sufrido una baja en cuanto al promedio de la rentabilidad sobre inversión puesto que bajó un $1 \%$, pasando de un $3 \%$ a un $2 \%$ entre los años 2017-2018. Esto sugiere que este tipo de universidades han perdido eficiencia.

\subsection{Análisis de las universidades públicas y privadas}

\section{a. Razón circulante}

En base a los resultados obtenidos, las universidades privadas han aumentado en gran cantidad su activo circulante, por lo cual, cada vez más poseen una mayor capacidad de saldar sus deudas de corto plazo. Esto se ve reflejado del 2017 al 2018, puesto que, en promedio, se obtuvo 3,13 veces y 5,46 veces, respectivamente (ver Tabla 6). 


\section{Tabla 6}

\section{Razón circulante: Comparación entre universidades públicas vs. privadas}

\begin{tabular}{lll}
\hline Universidades & Razón Circulante (2018) & Razón Circulante (2017) \\
\hline Universidades privadas & 24,73 & 1,01 \\
Universidad Internacional de la Rioja & 2,84 & 2,87 \\
Universidad a Distancia de Madrid & 0,52 & 0,91 \\
Universitat Oberta de Catalunya & & 1,41 \\
Universidad Internacional de Valencia & 3,50 & 1,83 \\
Universidad Isabel I & 3,77 & 3,74 \\
Universidad Europea Miguel de Cervantes & 0,44 & 0,78 \\
Universidad Nebrija & 6,28 & 7,90 \\
Universidad Camilo José Cela & 0,57 & 0,42 \\
Universidad Autónoma de Barcelona & 1,89 & 1,70 \\
Universidad Autónoma de Madrid & 15,35 & 14,50 \\
Universidad Alfonso X el Sabio & 0,14 & 0,53 \\
Universidad Católica San Antonio de Murcia & $\mathbf{5 , 4 6}$ & $\mathbf{3 , 1 3}$ \\
Promedio & & \\
Universidades públicas & 0,96 & 0,91 \\
Universidad Nacional de Educación a Distancia & 3,22 & 2,62 \\
Universidad de Alcalá & 0,61 & 0,24 \\
Universidad Pompeu Fabra & 1,28 & 1,38 \\
Universidad Complutense de Madrid & 2,29 & 0,83 \\
Universidad Rey Juan Carlos & $\mathbf{1 , 6 7}$ & $\mathbf{1 , 2 0}$ \\
Promedio & & \\
\hline
\end{tabular}

Fuente: Elaboración propia, 2021 en base a información publicada por las propias instituciones y datos obtenidos del Sistema de Análisis de Balances Ibéricos (SABI, 2020).

De acuerdo con las universidades públicas, se ve un aumento en la razón circulante al igual que en el caso anterior, aunque con menor proporción. Del 2017 al 2018 , el promedio de la ratio se incrementó de 1,20 veces a 1,67 veces, por lo cual se ve una mejoría en la capacidad de generar efectivo para el pago de las deudas de corto plazo. A diferencia de las universidades privadas, las universidades públicas han sido capaces de incrementar su liquidez sin obtener un exceso de efectivo que indique problemas de decisiones en cuanto a la inversión.

\section{b. Razón deuda total}

En el caso de las universidades privadas, en promedio, hubo una disminución del porcentaje de deuda, pasando de un $54 \%$ a un $47 \%$ del año 2017 al 2018 (ver Tabla 7). En ese sentido, necesitan menos financiamiento por parte de terceros $\mathrm{y}$, por consiguiente, se ven menos comprometidos con el pago de la deuda, manifestándose una situación favorable. 
Tabla 7

Razón deuda total: Comparación universidades públicas vs. privadas.

\begin{tabular}{lll}
\hline Universidades & Razón deuda total (2018) & Razón deuda total (2017) \\
\hline Universidades privadas & & \\
Universidad Internacional de la Rioja & 0,99 & 0,96 \\
Universidad a Distancia de Madrid & 0,06 & 0,93 \\
Universitat Oberta de Catalunya & 0,77 & 0,40 \\
Universidad Internacional de Valencia & & 0,62 \\
Universidad Isabel I & 0,06 & 0,43 \\
Universidad Europea Miguel de Cervantes & 0,21 & 0,20 \\
Universidad Nebrija & 0,72 & 0,73 \\
Universidad Camilo José Cela & 0,30 & 0,27 \\
Universidad Autónoma de Barcelona & 0,64 & 0,49 \\
Universidad Autónoma de Madrid & 0,39 & 0,39 \\
Universidad Alfonso X el Sabio & 0,06 & 0,06 \\
Universidad Católica San Antonio de Murcia & 0,99 & 0,99 \\
Promedio & $\mathbf{0 , 4 7}$ & $\mathbf{0 , 5 4}$ \\
Universidades públicas & & \\
Universidad Nacional de Educación a Distancia & 0,43 & 0,45 \\
Universidad de Alcalá & 0,17 & 0,12 \\
Universidad Pompeu Fabra & 0,59 & 0,95 \\
Universidad Complutense de Madrid & 0,67 & 0,69 \\
Universidad Rey Juan Carlos & 0,25 & 0,25 \\
Promedio & $\mathbf{0 , 4 2}$ & $\mathbf{0 , 4 9}$ \\
\hline & &
\end{tabular}

Fuente: Elaboración propia, 2021 en base a información publicada por las propias instituciones y datos obtenidos del Sistema de Análisis de Balances Ibéricos (SABI, 2020).

De modo análogo, en el caso de las universidades públicas también se ve una disminución del porcentaje de deuda, aunque de forma más leve pasando de un $49 \%$ a un $42 \%$ del 2017 al 2018, es decir, una disminución de 0,07 puntos porcentuales (ver Tabla 7). Por lo que, se puede pensar que este tipo de universidades no han sido capaces de disminuir de forma significativa el compromiso de pago con acreedores externos.

\section{c. Rotación de activos}

De acuerdo con los años 2017-2018, se obtuvo una leve mejoría en la utilización de activos totales para generar ventas por parte de las universidades privadas, pasando de un 0,84 a un 0,86 (ver Tabla 8 ). Esto es positivo debido a que existe un incremento de eficiencia en cuanto al uso de los activos totales adquiridos por la empresa y, por lo tanto, justifican la inversión realizada en su momento. 


\section{Tabla 8}

Rotación de activos: Comparación universidades públicas vs. privadas

\begin{tabular}{lcc}
\hline Universidades & Rotación de activos (2018) & Rotación de activos (2017) \\
\hline Universidades privadas & 0,97 & 0,88 \\
Universidad Internacional de la Rioja & 1,06 & 0,98 \\
Universidad a Distancia de Madrid & 1,26 & 1,48 \\
Universitat Oberta de Catalunya & & 0,59 \\
Universidad Internacional de Valencia & 0,81 & 0,77 \\
Universidad Isabel I & 0,83 & 0,76 \\
Universidad Europea Miguel de Cervantes & 1,51 & 1,62 \\
Universidad Nebrija & 0,66 & 0,58 \\
Universidad Camilo José Cela & 0,98 & 1,01 \\
Universidad Autónoma de Barcelona & 1,15 & 1,16 \\
Universidad Autónoma de Madrid & 0,21 & 0,21 \\
Universidad Alfonso X el Sabio & 0,001 & 0,001 \\
Universidad Católica San Antonio de Murcia & $\mathbf{0 , 8 6}$ & $\mathbf{0 , 8 4}$ \\
Promedio & & 0,82 \\
Universidades públicas & 0,84 & 0,43 \\
Universidad Nacional de Educación a Distancia & 0,44 & 0,58 \\
Universidad de Alcalá & 0,50 & 0,25 \\
Universidad Pompeu Fabra & 0,24 & 0,33 \\
Universidad Complutense de Madrid & 0,31 & $\mathbf{0 , 4 8}$ \\
Universidad Rey Juan Carlos & $\mathbf{0 , 4 7}$ & \\
Promedio &
\end{tabular}

Fuente: Elaboración propia, 2021 en base a información publicada por las propias instituciones y datos obtenidos del Sistema de Análisis de Balances Ibéricos (SABI, 2020).

En el caso de las universidades públicas, el caso es al revés, debido a que existe una leve disminución en la rotación de activos desde el año 2017 al 2018, donde pasó de un 0,48 a un 0,47 (ver Tabla 8), lo cual indica que este tipo de universidades han perdido eficiencia en la utilización de sus activos totales para generar ventas.

\section{d. Rentabilidad sobre el patrimonio (ROE)}

En el caso de las universidades privadas, el ROE tuvo un aumento significativo, en promedio, pasando de $26 \%$ a $160 \%$ (ver Tabla 9), por lo que se ha generado un mayor valor para los accionistas de este tipo de universidades $\mathrm{y}$, por consiguiente, las ha hecho más atractiva al otorgarle un mayor retorno a sus respectivos inversionistas, lo cual mejora la visión que tiene el mercado financiero de las mismas. 
Tabla 9

Rentabilidad sobre el patrimonio: Comparación universidades públicas vs. privadas

\begin{tabular}{lcr}
\hline Universidades & ROE (2018) & ROE (201 \\
\hline Universidades privadas & & 1,80 \\
Universidad Internacional de la Rioja & 13,16 & 0,005 \\
Universidad a Distancia de Madrid & 0,002 & $-0,03$ \\
Universitat Oberta de Catalunya & $-0,01$ & 0,31 \\
Universidad Internacional de Valencia & & 0,51 \\
Universidad Isabel I & 0,42 & 0,16 \\
Universidad Europea Miguel de Cervantes & 0,19 & 0,10 \\
Universidad Nebrija & 0,33 & 0,07 \\
Universidad Camilo José Cela & 0,12 & 0,01 \\
Universidad Autónoma de Barcelona & $-0,12$ & 0,03 \\
Universidad Autónoma de Madrid & $-0,01$ & 0,05 \\
Universidad Alfonso X el Sabio & 0,05 & 0,08 \\
Universidad Católica San Antonio de Murcia & 3,44 & $\mathbf{0 , 2 6}$ \\
Promedio & $\mathbf{1 , 6 0}$ & \\
Universidades públicas & & 0,07 \\
Universidad Nacional de Educación a Distancia & 0,03 & 0,062 \\
Universidad de Alcalá & 0,039 & 0,11 \\
Universidad Pompeu Fabra & 0,02 & 0,01 \\
Universidad Complutense de Madrid & $-0,01$ & 0,06 \\
Universidad Rey Juan Carlos & 0,02 & $\mathbf{0 , 0 6}$ \\
Promedio & $\mathbf{0 , 0 2}$ & \\
\hline
\end{tabular}

Fuente: Elaboración propia, 2021 en base a información publicada por las propias instituciones y datos obtenidos del Sistema de Análisis de Balances Ibéricos (SABI, 2020).

En el caso de las universidades públicas, el caso es distinto debido a que existe una disminución en el promedio del año 2017 y 2018, pasando de $6 \%$ a $2 \%$ (ver Tabla 9 ), es decir, un $4 \%$ menos que podría ser significativo para algunos inversionistas de este tipo de instituciones. Esto genera que las universidades públicas no sean tan atractivas a los ojos de los inversionistas, debido a que les otorga un menor retorno que antes.

\section{e. Rentabilidad sobre activos (ROA)}

En promedio, se puede observar que las universidades privadas han aumentado en un $3 \%$ el retorno de los activos, pasando de un 5\% a un $8 \%$ entre los años 2017-2018 (ver Tabla 10). Por lo cual, existe una leve mejora en la eficiencia con la cual este tipo de universidades utilizan los activos para generar utilidades. 
Tabla 10

Rentabilidad sobre activos: Comparación universidades públicas vs. Privadas

\begin{tabular}{lll}
\hline Universidades & $\mathbf{2 0 1 8}$ & $\mathbf{2 0 1 7}$ \\
\hline Universidades privadas & ROA \\
Universidad Internacional de la Rioja & 0,14 & 0,08 \\
Universidad a Distancia de Madrid & 0,002 & 0,005 \\
Universitat Oberta de Catalunya & $-0,003$ & $-0,01$ \\
Universidad Internacional de Valencia & & 0,12 \\
Universidad Isabel I & 0,33 & 0,13 \\
Universidad Europea Miguel de Cervantes & 0,15 & 0,13 \\
Universidad Nebrija & 0,09 & 0,03 \\
Universidad Camilo José Cela & 0,09 & 0,05 \\
Universidad Autónoma de Barcelona & $-0,04$ & 0,003 \\
Universidad Autónoma de Madrid & $-0,01$ & 0,03 \\
Universidad Alfonso X el Sabio & 0,05 & 0,09 \\
Universidad Católica San Antonio de Murcia & 0,03 & 0,001 \\
Promedio & $\mathbf{0 , 0 8}$ & $\mathbf{0 , 0 5}$ \\
Universidades públicas & & \\
Universidad Nacional de Educación a & 0,02 & 0,04 \\
Distancia & 0,032 & 0,054 \\
Universidad de Alcalá & 0,008 & 0,00 \\
Universidad Pompeu Fabra & $-0,01$ & 0,01 \\
Universidad Complutense de Madrid & 0,02 & 0,04 \\
Universidad Rey Juan Carlos & $\mathbf{0 , 0 1}$ & $\mathbf{0 , 0 3}$ \\
Promedio &
\end{tabular}

Fuente: Elaboración propia, 2021 en base a información publicada por las propias instituciones y datos obtenidos del Sistema de Análisis de Balances Ibéricos (SABI, 2020).

En el caso de las universidades públicas, el caso es al revés, debido a que el promedio de las instituciones en base al retorno sobre los activos, disminuyó de un $3 \%$ a $1 \%$ desde el 2017 al 2018, tal como se muestra en la Tabla 10. Por lo que, no es un buen indicio respecto a la gestión financiera que poseen este tipo de organizaciones educacionales debido a que la inversión en activos totales no se ve compensada con la cantidad de utilidades obtenidas.

\subsection{Desempeño financiero de universidades y su posición en los Rankings mundiales}

De acuerdo con la comparación entre universidades públicas y privadas, se obtuvo lo siguiente: Cabe destacar que tal como se puede evidenciar en la Tabla 11, no todas las universidades de los datos obtenidos figuran en los tres rankings que se utilizaron. Por lo que, el análisis se verá acotado al disminuir la cantidad de universidades disponibles para la comparación. 


\section{Tabla 11}

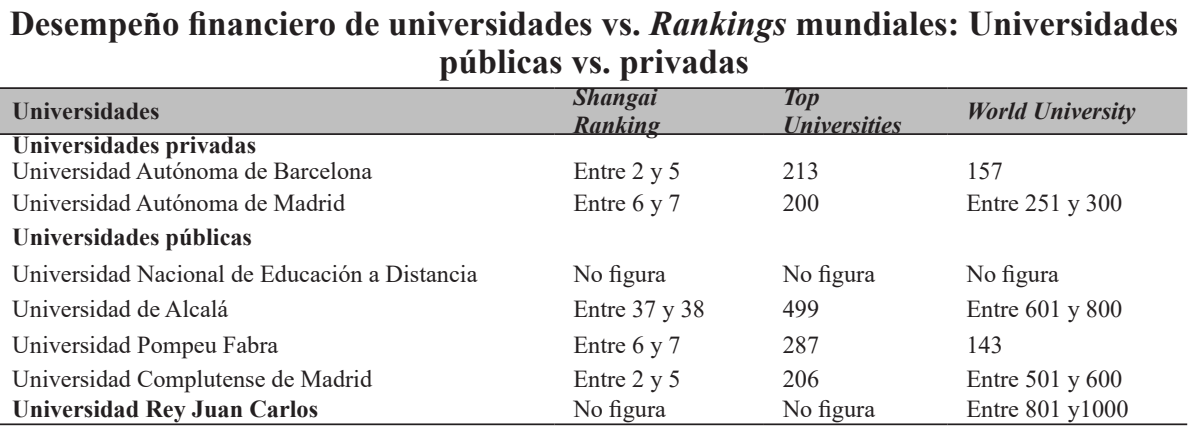

Fuente: Elaboración propia, 2021 en base a información obtenida directamente de los sitios de cada ranking.

Como se puede observar, existe una mayor cantidad de universidades públicas que figuran dentro de los rankings internacionales, aunque con una menor posición que las organizaciones educacionales privadas. Dentro de las diferencias, en base a los datos encontrados, en el caso de las universidades públicas, poseen un menor porcentaje de deuda respecto a las universidades privadas. Así como también, tienen una mayor capacidad de generar efectivo para pagar las deudas de corto plazo. Por lo que, las empresas privadas a pesar de tener mejores puestos no tienen mejor rendimiento en lo que respecta a la ratio deuda total y razón circulante. Por otra parte, las diferencias que se pueden encontrar entre universidades con distintas modalidades de acuerdo con su oferta académica se reflejan en la Tabla 12.

\section{Tabla 12}

\section{Desempeño financiero de universidades vs. Rankings mundiales: Universidades presenciales / online versus Universidades con modalidad presencial}

\begin{tabular}{|c|c|c|c|}
\hline Universidades & Shangai Ranking & Top Universities & World University \\
\hline \multicolumn{4}{|l|}{ Universidades presenciales / online } \\
\hline Universidad Autónoma de Barcelona & Entre 2 y 5 & 213 & 157 \\
\hline Universidad Autónoma de Madrid & Entre 6 y 7 & 200 & Entre 251 y 300 \\
\hline \multicolumn{4}{|l|}{ Universidades presenciales } \\
\hline Universidad de Alcalá & Entre 37 y 38 & 499 & Entre 601 y 800 \\
\hline Universidad Pompeu Fabra & Entre 6 y 7 & 287 & 143 \\
\hline Universidad Complutense de Madrid & Entre 2 y 5 & 206 & Entre 501 y 600 \\
\hline Universidad Rey Juan Carlos & No figura & No figura & Entre 801 y 1000 \\
\hline
\end{tabular}

Fuente: Elaboración propia, 2021 en base a información obtenida directamente de los sitios de cada ranking.

En este caso, ninguna universidad $100 \%$ online figuraba en alguno de los tres rankings analizados. Por lo cual, internacionalmente no serían tan bien reconocidas como las universidades presenciales/online o puramente presencial. Las universidades presenciales, poseen una mayor cantidad de universidades que se encuentran en los rankings, pero con una menor posición que las universidades con modalidad presencial/online.

Por otra parte, las diferencias significativas en base a las ratios obtenidas en este estudio corresponden a un mayor resultado en la razón circulante y un menor porcentaje de deuda en la estructura de capital de este tipo de universidades. Por lo que, las universidades 
Barrientos Oradini, Nicolás Pablo; Vicencio-Ríos, Gustavo; Armijos Armijos, Juan Carlos y González Navarro, Yuri Christian

Desempeño de Universidades y Escuelas de Negocios: Instituciones españolas presenciales y a Distancia

presenciales tienen una mayor capacidad de generar efectivo para pagar deudas a corto plazo y depender menos de financiamiento de acreedores externos.

\subsection{Escuelas de negocios}

Descriptivamente, las escuelas de negocios con modalidad online disminuyeron su capacidad de generar efectivo a corto plazo, para saldar sus obligaciones con terceros desde el año 2017 al 2018, pasando de 2,60 veces a 2,18 veces (ver Tabla 13). Esta disminución de la ratio financiera no indicaría un escenario tan negativo, debido a que el ideal es que dé como resultado uno, como máximo dos.

Tabla 13

Comparación escuelas de negocios presencial versus presencial/online vs. online

\begin{tabular}{lcc}
\hline Escuelas de Negocio & $\mathbf{2 0 1 8}$ & $\mathbf{2 0 1 7}$ \\
\cline { 3 - 3 } & Razón circulante & Razón circulante \\
\hline Escuelas de Negocio online & & \\
ISDI Business School & 1,28 & 1,38 \\
IEBS Business School & 1,24 & 1,14 \\
ENYD Business School & 0,92 & 1,22 \\
AUCAL Business School & 5,26 & 6,67 \\
Promedio & $\mathbf{2 , 1 8}$ & $\mathbf{2 , 6 0}$ \\
Escuelas de Negocio Presencial / Online & & 1,06 \\
EUDE Business School & 1,08 & 0,92 \\
IMF Business School & 0,79 & 0,91 \\
ESERP Business School & 0,87 & $\mathbf{0 , 9 6}$ \\
Promedio & $\mathbf{0 , 9 1}$ & 1,42 \\
Escuelas de Negocio Presencial & & 2,68 \\
EAE Business School & 1,33 & 0,99 \\
IESIDE Business School & 0,00 & 1,47 \\
CESMA Business School & 0,48 & $\mathbf{1 , 6 4}$ \\
EDEM Business School & 0,00 & $\mathbf{0 , 4 5}$ \\
Promedio & & \\
\hline
\end{tabular}

Fuente: Elaboración propia, 2021 en base a información publicada por las propias instituciones y datos obtenidos del Sistema de Análisis de Balances Ibéricos (SABI, 2020).

Las escuelas de negocios con ambas modalidades tuvieron una leve disminución en su capacidad de generar efectivo para pagar sus deudas de corto plazo con terceros, pasando de un 0,96 a un 0,91 veces desde el año 2017 al 2018 (ver Tabla 13). Esta disminución de la ratio financiera no indicaría un escenario tan negativo, debido a que el ideal es que dé como resultado uno y los números promedio de este tipo de instituciones financieras no se alejan tanto del ideal.

Por el lado de las escuelas de negocios presenciales, en promedio, sufrieron una considerable caída en la ratio razón circulante, debido a que del 2017 a 2018, el número pasa 1,64 a 0,45 veces, tal como se puede apreciar en la Tabla 13. Esto indica que la gestión financiera de este tipo de organizaciones ha sido a todas luces errónea.

\section{a. Razón deuda total}

Del 2017 al 2018, la razón de deuda total que poseen las escuelas de negocios online ha disminuido de $63 \%$ a $49 \%$ (ver Tabla 14), lo cual es un escenario favorable al disminuir el compromiso que este tipo de organizaciones poseen con terceros $\mathrm{y}$, por consiguiente, podrían apoyarse en mayor cantidad de su financiamiento interno, es decir, de sus accionistas actuales. 
Tabla 14

Deuda total: Comparación escuelas de negocios presencial versus presencial/ online vs. Online

\begin{tabular}{lcc}
\cline { 1 - 1 } Escuelas de Negocio & Razón deuda total & Razón deuda total \\
\cline { 1 - 1 } Escuelas de Negocio online & & \\
ISDI Business School & 0,21 & 0,86 \\
IEBS Business School & 0,80 & 0,87 \\
ENYD Business School & 0,75 & 0,65 \\
AUCAL Business School & 0,19 & 0,15 \\
Promedio & $\mathbf{0 , 4 9}$ & $\mathbf{0 , 6 3}$ \\
Escuelas de Negocio presencial / online & 0,96 & 0,93 \\
EUDE Business School & 0,66 & 0,66 \\
IMF Business School & 0,59 & 0,55 \\
ESERP Business School & $\mathbf{0 , 7 4}$ & $\mathbf{0 , 7 1}$ \\
Promedio & & 0,69 \\
Escuelas de Negocio presencial & 0,74 & 0,37 \\
EAE Business School & 0,00 & 0,77 \\
IESIDE Business School & 2,38 & 0,93 \\
CESMA Business School & 0,00 & $\mathbf{0 , 6 9}$ \\
EDEM Business School & $\mathbf{0 , 7 8}$ & \\
Promedio & & \\
\hline
\end{tabular}

Fuente: Elaboración propia, 2021 en base a información publicada por las propias instituciones y datos obtenidos del Sistema de Análisis de Balances Ibéricos (SABI, 2020).

A diferencia del caso anterior, las escuelas de negocios que poseen ambas modalidades han sufrido un leve aumento de su razón deuda total, pasando de un $71 \%$ a un 74\% desde el 2017 al 2018 (ver Tabla 14). Respecto a las escuelas de negocios con modalidad presencial, del 2017 al 2018 se vio incrementada la razón deuda total de un $78 \%$ a un $69 \%$, por lo que este tipo de organizaciones han podido necesitar una mayor cantidad de financiamiento por parte de terceros, lo cual genera un mayor compromiso de pago con estos.

\section{b. Rotación de activos}

Del 2017 al 2018, las escuelas de negocios con modalidad online han experimentado una leve disminución en su rotación de activos, es decir, ha disminuido la eficiencia con la cual se utilizan los activos para generar ventas, pasando de un 1,66 a un 1,60 (ver Tabla 15).

Tabla 15

Rotación de activos: Comparación escuelas de negocios presencial versus presencial/online vs. online

\begin{tabular}{|c|c|c|}
\hline \multirow{2}{*}{ Escuelas de Negocio } & 2018 & 2017 \\
\hline & Razón deuda total & Razón deuda total \\
\hline \multicolumn{3}{|l|}{ Escuelas de Negocio online } \\
\hline ISDI Business School & 1,26 & 1,39 \\
\hline IEBS Business School & 1,72 & 1,69 \\
\hline ENYD Business School & 2,13 & 1,70 \\
\hline AUCAL Business School & 1,30 & 1,87 \\
\hline Promedio & 1,60 & 1,66 \\
\hline Escuelas de Negocio presencial / online & & \\
\hline
\end{tabular}


Barrientos Oradini, Nicolás Pablo; Vicencio-Ríos, Gustavo; Armijos Armijos, Juan Carlos y González Navarro, Yuri Christian

Desempeño de Universidades y Escuelas de Negocios: Instituciones españolas presenciales y a Distancia

\begin{tabular}{lcc}
\multicolumn{2}{c}{ Cont... Tabla 15 } \\
\hline EUDE Business School & & \\
IMF Business School & 1,12 & 1,02 \\
ESERP Business School & 1,19 & 1,22 \\
Promedio & 2,24 & 2,75 \\
Escuelas de Negocio presencial & $\mathbf{1 , 5 2}$ & $\mathbf{1 , 6 7}$ \\
EAE Business School & & \\
IESIDE Business School & 0,75 & 0,65 \\
CESMA Business School & 0,00 & 0,00 \\
EDEM Business School & 1,66 & 1,03 \\
Promedio & 0,00 & 0,24 \\
\hline
\end{tabular}

Fuente: Elaboración propia, 2021 en base a información publicada por las propias instituciones y datos obtenidos del Sistema de Análisis de Balances Ibéricos (SABI, 2020).

De acuerdo con las escuelas de negocios con ambas modalidades, es decir, presencial y online, la rotación de activos que poseen del 2017 a 2018 ha experimentado una baja, pasando de 1,67 a 1,53 . Por su parte, las escuelas de negocios de tipo presencial, del 2017 al 2018, han visto aumentada su rotación de activos pasando de un 0,48 a un 0,60 (ver Tabla 15), lo cual demuestra que han sido capaces de aumentar la eficiencia con la cual utilizan los activos para generar ventas $\mathrm{y}$, por consiguiente, han sido capaces de atraer una mayor cantidad de estudiantes que quieran tener esta modalidad de estudio, lo cual se traduce en una mayor cantidad de ingresos.

\section{c. Rentabilidad sobre el patrimonio (ROE)}

De acuerdo con la rentabilidad sobre el patrimonio de las escuelas de negocios online, del año 2017 al 2018 han experimentado un incremento de 0,22 a 0,44 , tal como se evidencia en la Tabla 16; por lo que este tipo de organizaciones han sido capaces de generar una mayor cantidad de utilidades gracias al uso de la inversión por parte de los accionistas.
Tabla 16

Rentabilidad sobre el patrimonio: Comparación escuelas de negocios presencial vs. presencial/online vs. online

\begin{tabular}{|c|c|c|}
\hline \multirow{2}{*}{ Escuelas de Negocio } & 2018 & 2017 \\
\hline & ROE & ROE \\
\hline \multicolumn{3}{|l|}{ Escuelas de Negocio online } \\
\hline ISDI Business School & 0,14 & $-0,62$ \\
\hline IEBS Business School & 0,60 & 0,30 \\
\hline ENYD Business School & 0,82 & 0,89 \\
\hline AUCAL Business School & 0,18 & 0,31 \\
\hline Promedio & 0,44 & 0,22 \\
\hline \multicolumn{3}{|c|}{$\begin{array}{l}\text { Escuelas de Negocio Presencial } \\
\text { / Online }\end{array}$} \\
\hline EUDE Business School & 0,81 & 0,37 \\
\hline IMF Business School & 0,17 & 0,09 \\
\hline ESERP Business School & 0,78 & 0,60 \\
\hline Promedio & 0,58 & 0,35 \\
\hline \multicolumn{3}{|c|}{ Escuelas de Negocio Presencial } \\
\hline EAE Business School & 0,199 & 0,743 \\
\hline IESIDE Business School & 0,00 & 0,00 \\
\hline CESMA Business School & 0,02 & 007 \\
\hline EDEM Business School & 0,00 & $-0,01$ \\
\hline Promedio & 0,05 & 0,20 \\
\hline
\end{tabular}

Fuente: Elaboración propia, 2021 en base a información publicada por las propias instituciones y datos obtenidos del Sistema de Análisis de Balances Ibéricos (SABI, 2020).

Las escuelas de negocios que poseen 
modalidad presencial/ online, han aumentado su rentabilidad sobre el patrimonio de un 0,35 a un 0,58 del año 2017 al 2018 (ver Tabla 16), lo cual es positivo para los accionistas de este tipo de instituciones debido a que obtienen una mayor cantidad de ganancia gracias al aumento de utilidades que posee la organización debido al uso óptimo del capital contable otorgado. Un caso inverso ha ocurrido con la modalidad presencial que ha descendido de 0,20 a 0,05 en el mismo período temporal mostrando un efecto negativo.

\section{d. Rentabilidad sobre la inversión}

Las escuelas de negocios online, en un año, han sido capaces de aumentar la rentabilidad que obtienen sobre su inversión, por lo que consiguen una mayor cantidad de utilidades al usar con mayor eficiencia sus activos totales, debido a pasar de 0,14 a 0,17 del 2017 al 2018 (ver Tabla 17).

Tabla 17

Rentabilidad sobre la inversión: Comparación escuelas de negocios presencial vs. presencial/online vs. online

\begin{tabular}{lll}
\hline Escuelas de Negocio & $\mathbf{2 0 1 8}$ & $\mathbf{2 0 1 7}$ \\
\cline { 1 - 3 } Escuelas de Negocio online & ROA & ROA \\
ISDI Business School & 0,01 & $-0,09$ \\
IEBS Business School & 0,32 & 0,04 \\
ENYD Business School & 0,21 & 0,31 \\
AUCAL Business School & 0,14 & 0,31 \\
Promedio & $\mathbf{0 , 1 7}$ & $\mathbf{0 , 1 4}$ \\
Escuelas de Negocio presencial / online & & \\
EUDE Business School & 0,03 & 0,02 \\
IMF Business School & 0,06 & 0,03 \\
ESERP Business School & 0,32 & 0,27 \\
Promedio & $\mathbf{0 , 1 4}$ & $\mathbf{0 , 1 1}$ \\
Escuelas de Negocio presencial & & \\
EAE Business School & 0,052 & 0,230 \\
IESIDE Business School & 0,00 & 0,05 \\
CESMA Business School & $-0,03$ & 0,02 \\
EDEM Business School & 0.01 & $-0,01$ \\
Promedio & $\mathbf{0 , 0 1}$ & $\mathbf{0 , 0 7}$ \\
\hline
\end{tabular}

Fuente: Elaboración propia, 2021 en base a información publicada por las propias instituciones y datos obtenidos del Sistema de Análisis de Balances Ibéricos (SABI, 2020).

De acuerdo con las escuelas de negocios con ambas modalidades, la rentabilidad sobre la inversión ha presentado una leve mejoría, pasando de un 0,11 a un 0,14 del 2017 al 2018, tal como se evidencia en la Tabla 17. En conclusión, este tipo de organizaciones está obteniendo buenos resultados al momento de utilizar sus activos totales para generar utilidades. Por su parte, en base a las escuelas de negocios presenciales, del año 2017 a 2018, la rentabilidad sobre los activos ha disminuido de 0,07 a 0,01, lo cual significaría que, en un año, este tipo de organizaciones ha perdido eficiencia con la cual utiliza los activos para poder generar utilidades.

Estos resultados hallados en la presente investigación, se producen en un contexto en el cual en los últimos años las Escuelas de Negocios se encuentran operando en un mercado altamente competitivo (Jain et al., 2013). La explicación reside en que el sector en que participan presenta rasgos similares a las 
de una industria de servicio (Danjuma y Rasli, 2013). En este escenario, las instituciones de educación superior -y sobre todo las Escuelas de Negocios- deben innovar constantemente y responder a los cambios sociales, así como a las nuevas dinámicas competitivas (ArayaCastillo, 2015).

Por otra parte, las instituciones de educación superior, especialmente las que operan con capacidades crecientes, tienen la problemática de servir a un mercado cada vez mayor de alumnos, más heterogéneo social y culturalmente (Silvio, 1998). En este contexto, las Escuelas de Negocios, deben reconocer como una oportunidad de mercado, la tendencia internacional que existe hacia una población estudiantil más diversificada (Archer, Hutchings y Ross, 2003). Esto se puede fundar en la necesidad de responder a las nuevas directrices del sector industrial por medio del diseño de programas de formación que hagan uso de las tecnologías de la información (Buil et al., 2012).

Además, la calidad en las escuelas de negocios es un tema muy atingente en la actualidad, asociado a hacer frente a la exigencia estudiantil y a la elevada competencia. Por ello, resulta fundamental orientar los esfuerzos en adoptar estrategias de marketing dirigidas a mejorar la imagen y percepción de estas instituciones, así como, es necesario que las escuelas dispongan o estén sometidas a adecuados instrumentos de medición y monitoreo de la calidad.

\section{Conclusiones}

El principal aporte de este estudio reside en que, a través de un análisis de desempeño financiero de las Universidades y Escuelas de Negocio en España, se alcanza a comprender (al menos en parte) el comportamiento de las instituciones comparando públicas y privadas; presenciales y virtuales; en función además de los principales rankings internacionales de educación superior.

Entre los diferentes hallazgos del trabajo se encuentran, primero, a pesar que, en base a los rankings, las universidades públicas poseen una mayor cantidad de ellas posicionadas dentro de estos, las universidades privadas ostentan un mejor posicionamiento que las universidades públicas. La explicación radica en que las universidades privadas superan en creces el desempeño financiero que poseen las universidades públicas puesto que, en la mayoría de las ratios financieras analizadas, el promedio de las privadas supera a las públicas.

Además, las universidades privadas han sido capaces de obtener mejores resultados financieros que las universidades públicas, gracias a que son capaces de generar una mayor cantidad de efectivo para pagar sus deudas de corto plazo, así como utilizan de forma más eficiente sus activos tanto para generar ventas como utilidades.

En segundo lugar, las universidades con modalidad presencial/online superan con creces a las universidades online y a las que son solamente presenciales, debido a que poseen una mayor eficiencia al momento de utilizar sus activos tanto para generar ventas, es decir atraer más alumnos y, por consiguiente, matrículas. También, generan más utilidades que ayudan a obtener liquidez por su propia cuenta, para de ese modo ir disminuyendo tanto el financiamiento externo como interno. Es por ello que, dentro de esta categoría de universidades, poseen un mayor y mejor desempeño financiero de acuerdo a su competencia.

Por otra parte, las universidades presenciales no destacan dentro del desempeño financiero en base a las ratios analizadas, lo cual podría demostrar que, a pesar de ser la modalidad preferida por los estudiantes, no quiere decir que sean mejor administradas financieramente. Por último, las universidades online, a través del tiempo, están mejorando su posición dentro de la competencia y así eliminan cada vez más el estigma que se tiene sobre ellas.

En tercer término, en base a la comparación de escuelas de negocios online versus presenciales, se puede decir que, a pesar de ser ambas privadas, las escuelas online poseen un mayor rendimiento financiero 
que las presencial/online y las puramente presenciales, debido a que poseen, en promedio, una mayor eficiencia al momento de utilizar los activos (situación revelada tanto en el 2017 como en el 2018).

Por otra parte, la capacidad de generar efectivo para saldar sus pasivos de corto plazo también fue sustancialmente superior al promedio de las otras escuelas de negocios $\mathrm{y}$, a su vez, son el tipo de instituciones que mejor utilizan sus activos totales para generar utilidades que les otorguen valor a las organizaciones. Por lo tanto, esto demuestra que, a pesar del prejuicio que se tiene en base a la calidad y posición financiera del tipo de enseñanza online, este tipo de escuela de negocios muestran una mejor capacidad de desempeñarse financieramente dentro de la industria educacional.

Finalmente, los rankings como indicadores de calidad pueden servir para apreciar diferencias entre instituciones, donde en general se esperaría evidenciar que las universidades y escuelas de negocio que estén posicionadas en los rankings internacionales posean también un desempeño financiero superior al promedio. Sin embargo, quedó demostrado que el desempeño financiero en el caso de las universidades privadas no tiene mayor repercusión ni relación con la posición obtenida en los rankings como un referente de calidad. De manera análoga, lo mismo sucede con las instituciones públicas.

Por último, es importante indicar que ninguna de las universidades bajo la modalidad $100 \%$ online figura en alguno de los tres rankings analizados, por lo cual es posible concluir que estas aún no logran consolidar el posicionamiento en cuanto a calidad en comparación a las instituciones de educación tradicional.

Sin embargo, pese a obtener algunas respuestas a su propósito, el trabajo presentó algunas limitaciones. La más notable de ellas fue la información parcial a la que se pudo acceder representando solamente una porción del más vasto universo total de universidades y escuelas de negocios. Ampliar en un futuro el espectro, resulta una opción interesante. Además, otra gran la limitación ha resultado asociar, o peor aún emular, calidad a desempeño financiero.

El trabajo prueba una relación estrecha, o mejor aún, que un buen desempeño financiero es una condición necesaria pero no suficiente para sobresalir en calidad. De este modo, la hipótesis del estudio se cumple solo parcialmente, evidenciando que la calidad en la educación superior obedece a múltiples variables (no tratadas en el estudio) más allá de disponer de buenos programas en base a metas de ahorro, con un desempeño financiero favorable que logre impactar como atributo relevante en la decisión de inversión, generando también beneficios cuantificables para la oferta y la demanda.

Justamente, una futura línea de investigación pasará por precisar cuál es rol concreto de la economía y las finanzas en materia de calidad de educación superior, además de indagar sobre qué otras variables pueden incidir en este concepto.

\section{Notas}

1 Los Estados Financieros utilizados para el cálculo de datos fueron obtenidos del Sistema SABI (Sistema de Análisis de Balances Ibéricos), que analiza los balances de más de 2,7 millones de empresas españolas, disponible en https://sabi.bvdinfo.com. Por otra parte, los datos financieros de las siguientes universidades fueron obtenidos directamente en los sitios $W e b$ institucionales de cada una de ellas: Universidad Nacional de educación a Distancia, Universitat Oberta de Catalunya, Universidad Internacional de Valencia, Universidad Autónoma de Barcelona, Universidad Autónoma de Madrid, Universidad de Alcalá, Universidad Pompeu Fabra, Universidad Católica San Antonio de Murcia, y Universidad Rey Juan Carlos.

${ }^{2}$ Es importante señalar que los rankings a utilizar son internacionales y corresponden al "Shangai Ranking", "Top Universities" y "World university". Por otra parte, antes de realizar la interpretación de los datos, cabe 
Barrientos Oradini, Nicolás Pablo; Vicencio-Ríos, Gustavo; Armijos Armijos, Juan Carlos y González Navarro, Yuri Christian

Desempeño de Universidades y Escuelas de Negocios: Instituciones españolas presenciales y a Distancia

destacar que la Universidad Internacional de Valencia, EDEM Business School e IESIDE Business School, no tienen información financiera del año 2018, puesto que públicamente, los estados financieros llegan hasta el $2017 \mathrm{y}$, al analizar a las organizaciones en conjunto, se tomará como referencia los resultados de las ratios como la media entre ellas, para verificar su tendencia en los años 2017-2018 y luego, comparar entre tipo de entidad de educación superior.

\section{Referencias bibliográficas}

\section{Alstete, J. W. (1995). Benchmarking in Higher} Education: Adapting best practices to improve quality. ASHE-ERIC Higher Education Report, No. 5. The George Washington Graduate School of Education and Human Development. https://eric.ed.gov/?id=ED402801

Altbach, P. G., Reisberg, L., y Rumbley, L. E. (2009). Trends in global higher education: Tracking an academic revolution. UNESCO, World Conference on Higher Education. http://www.cep.edu.rs/public/ Altbach, Reisberg, Rumbley Tracking an Academic Revolution, UNESCO 2009.pdf

Arambewela, R., y Hall, J. (2006). A comparative analysis of international education satisfaction using SERVQUAL. Journal of Services Research, 6(E). 141-163.

Araya, L. (2014). Propuesta de metodología en la determinación de los grupos estratégicos. Revista Escuela de Administración de Negocios, (76), 66-77. https:// doi.org/10.21158/01208160. $\underline{\mathrm{n} 76.2014 .798}$

Araya-Castillo, L. (2015). Dinámica competitiva de las universidades en Chile y la necesidad de potenciar el mercado de educación a distancia. Aposta. Revista de Ciencias Sociales, (64), 1-30.

Archer, L., Hutchings, M., y Ross, A. (2003). Higher education and social class: Issues of exclusion and inclusion. Routledge.

Area, M., y Adell, J. (2009). E-Learning: Enseñar y aprender en espacios virtuales. En J. De Pablos (Coord.), Tecnología educativa: La formación del profesorado en la era de Internet (pp. 391-424). Ediciones Aljibe.

Bernal, C. A. (2010). Metodología de la investigación: Administración, economía, humanidades y ciencias sociales. Pearson Educación.

Brunner, J. J. (2006). Sistema privatizado y mercados universitarios: Competencia reputacional y sus efectos. Informe elaborado en el marco del proyecto FONDECYT No. 1050138. http://200.6.99.248/ bru487cl/files/ ESPrv\%26Mer.pdf

Buil, I., Hernández, B., Sese, F. J., y Urquizu, P. (2012). Los foros de discusión y sus beneficios en la docencia virtual: Recomendaciones para un uso eficiente. Innovar, 22(43), 131-143.

Cabero, J. (2004). La transformación de los escenarios educativos como consecuencia de la aplicación de las TICs: Estrategias educativas. En M. Vera y D. Pérez (Eds.), Formación de la ciudadanía: Las TICs y los nuevos problemas (pp.17-43). Asociación Universitaria de profesores de Didáctica de las Ciencias Sociales.

Crespo-Fajardo, J. L., y Pillacela-Chin, L. (2021). Nuevas tecnologías en los primeros subniveles de Educación Cultural y Artística en Ecuador. Revista de Ciencias Sociales (Ve), XXVII(1), 334-346. https://doi.org/10.31876/rcs. v27i1.35319 
Danjuma, I., y Rasli, A. (2013). Higher Education and Knowledge Economy: A Focus on Nigeria. Jurnal Teknologi, 64(3), 87-91. https://doi.org/10.11113/ it.v64.2275

Denegri, M., Cabezas, D., Novoa, M., Peralta, J., y Estrada, C. (2013). Personalidad de marca en carreras de psicología de zonas territoriales extremas: Arica y Punta Arenas. Magallania, 41(2), 85100.

Dill, D. (2010). Quality assurance in higher education: Practices and issues. In P. Peterson, E. Baker y B. McGaw (Eds.), International Encyclopedia of Education (pp. 377-383). Elsevier. https://doi.org/10.1016/B978-0-08044894-7.00833-2

Gómez, J. (2017). Interacciones MoodleMOOC: Presente y futuro de los modelos de e-learning y b-learning en los contextos universitarios. EccoS - Revista Cientifica, (44), 241257. https://doi.org/10.5585/eccos. $\underline{\mathrm{n} 44.7353}$

Hinojosa, C. A., Epiquién, M., y Morante, M. A. (2021). Entornos virtuales como herramienta de apoyo al sistema de aprendizaje contable: Un desarrollo necesario. Revista de Ciencias Sociales (Ve), XXVII(E-3), 64-75. https://doi. org/10.31876/rcs.v27i.36489

Jain, R., Sahney, S., y Sinha, G. (2013). Developing a scale to measure students' perception of service quality in the Indian context. The TQM Journal, 25(3), 276-294. https://doi. org/10.1108/17542731311307456

Jain, R., Sinha, G., y Sahney, S. (2011). Conceptualizing service quality in higher education. Asian Journal on Quality, 12(3), 296-314. https://doi. org/10.1108/15982681111187128

Kells, H. R. (1992). Preformance indicators for higher education: A critical review with policy recommendations. Document No. PHREE/92/56. World Bank. https://documents1.worldbank. org/curated/en/430761468766509535/ pdf/multi-page.pdf

Macau, R. (2004). TIC: ¿PARA QUÉ? (Funciones de las tecnologías de la información y la comunicación en las organizaciones). Revista de Universidad y Sociedad del Conocimiento (RUSC), 1(1), 1-12.

Maftum, M., y Batista, J. (2008). Capacitaçao pedagógica na modalidade de Educaçao a distancia: Desafio para ativar procesos de mudança na formaçao de prodissionais de saúde. Cogitare Enfermagem, 13(1), 132139. $\quad$ https://dx.doi.org/10.5380/ ce.v13i1.11973

Martí, J. A. (2011). Aprendizaje mezclado (B-Learning) Modalidad de formación de profesionales. Revista Universidad EAFIT, 45(154), 70-77.

Morán, F. E., Morán, F. L., Morán, F. J., y Sánchez, J. A. (2021). Tecnologías digitales en las clases sincrónicas de la modalidad en línea en la Educación Superior. Revista de Ciencias Sociales (Ve), XXVII(3), 317- 333. https://doi. org/10.31876/rcs.v27i3.36772

Pinto, M. (1992). El resumen documental: Principios y métodos. Pirámide.

Rodríguez, A., y Pérez, A. O. (2017). Métodos científicos de indagación y de construcción del conocimiento. Revista Escuela de Administración de Negocios, (82), 179-200. https:// doi.org/10.21158/01208160. $\underline{\mathrm{n} 82.2017 .1647}$

Sangrá, A. (2001). Enseñar y aprender en la virtualidad. Educar, (28), 117-131. https://raco.cat/index.php/Educar/ article/view/20746

Silvio, J. (1998). La virtualización de la educación superior: alcances, 
Barrientos Oradini, Nicolás Pablo; Vicencio-Ríos, Gustavo; Armijos Armijos, Juan Carlos y González Navarro, Yuri Christian

Desempeño de Universidades y Escuelas de Negocios: Instituciones españolas presenciales y a Distancia

posibilidades y limitaciones. Educación Superior y Sociedad, 9(1), 27-50.

Smith, M. (2001). Writing a successful paper. The Trey Research Monthly, 53, 149150.

\section{Valverde-Berrocoso,} Herramientas de comunicación sincrónica y asincrónica. En J. I.
Aguaded y J. Cabero (Dirs.), Educar en red: Internet como recurso para la educación (pp. 57-81). Ediciones Aljibe.

Varguillas, C. S., y Bravo, P. C. (2020). Virtualidad como herramienta de apoyo a la presencialidad: Análisis desde la mirada estudiantil. Revista de Ciencias Sociales (Ve), XXVI(1), 219-232. https://doi.org/10.31876/rcs. v26i1.31321 